\title{
LIGHT-ASSISTED TRANSPORT OF NITRIC OXIDE ACROSS MEMBRANES CONTAINING NITROSYL CHLORIDE SOLUTIONS
}

\author{
RAVI JAIN \\ The BOC Group Inc., Group Technical Center, Murray Hill, NJ 07974 (U.S.A.) \\ and JEROME S. SCHULTZ*
}

Department of Chemical Engineering, The University of Michigan, Ann Arbor, MI 48109 (U.S.A.)

(Received October 21, 1985; accepted November 25, 1985)

\section{Summary}

Experimental evidence for the light-assisted concentration of nitric oxide taking part in a reversible photochemical reaction in a solution is presented. For the system, nitrosyl chloride-nitric oxide, a gradient of light energy across the membrane sandwich containing nitrosyl chloride solution results in a gradient of nitric oxide concentration across the membrane. For the experimental conditions employed, nitric oxide concentration ratios of up to 15 were obtained. The experimental results are explained using an "equilibrium-facilitation" model. The photodiffusion membrane configuration of the kind discussed can be used for the chemical storage of solar energy.

\section{Introduction}

The decomposition of nitrosyl chloride has been studied for a long time as a possible system for the chemical storage of solar energy [1-7]. The attractiveness of the system lies in the fact that it absorbs a significant amount of energy in the visible range of solar spectrum, has a high quantum yicld of 2 for the formation of nitric oxide, has a significant free energy change $(4.9 \mathrm{kcal} / \mathrm{mol}$ of NOCl) and a high heat of reaction $(9.0 \mathrm{kcal} / \mathrm{mol}$ of $\mathrm{NOCl})$. The free energy change can be utilized to recover energy stored in the products of decomposition (nitric oxide and chlorine) by combining them in a fuel cell, while the heat of reaction can be utilized by combining the products in a heat exchanger.

The decomposition products recombine fairly rapidly upon the removal of light energy in both gaseous and liquid systems. Therefore, most of the past work on using this reaction for energy conversion has involved devising better schemes for separating the products before they have had a chance to recombine. For this purpose both static and flow photo-reactors have been proposed. In the static system [4-6], an organic solution of nitrosyl

*To whom correspondence should be addressed. 
chloride is illuminated and the nitric oxide formed in the gas phase is collected. For this purpose a solvent such as $\mathrm{CCl}_{4}$, which dissolves $\mathrm{NOCl}$ and $\mathrm{Cl}_{2}$ to a significant degree but nitric oxide only very slightly, was used. The quantum yields in the static systems decrease with time because of the product build up. To overcome these problems, flow systems have been utilized where a stream containing nitrosyl chloride is illuminated and the nitric oxide formed is continuously removed [4, 8]. In both these approaches, the amount of $\mathrm{NOCl}$ decomposed is less than $5 \%$. McKee et al. $[7,9]$ carried out the decomposition of $\mathrm{NOCl}$ in the gas phase, and used a solvent to remove NO from the illuminated gas space. The product yields were higher than $5 \%$, but a liquid phase decomposition is preferable because in solution, a large amount of NOCl can be concentrated in a small volume.

In this paper, we present a different approach for the utilization of the $\mathrm{NOCl}$ decomposition reaction. The approach involves the differential illumination of a membrane containing nitrosyl chloride solution. The experimental procedure is similar to the one discussed by Schultz [10] for the carbon monoxide-hemoglobin system. The experiments discussed in this paper produced a concentration difference of nitric oxide across the membrane. This concentration difference can be used to run a fuel cell for the chemical storage of solar energy [9]. The theory for the transport in a photodiffusion membrane discussed in a previous paper [11] was used to analyze the results. The effects of nitric oxide concentration, nitroxyl chloride concentration and the light energy on transport of nitric oxide were investigated.

\section{Theory}

The analysis of diffusion and reaction in a photomembrane configuration developed by Jain and Schultz [11] is the basis of the approach used here. The stoichiometry of the NOCl decomposition reaction is the same in light or in dark and is given by eqn. (1). The decomposition rate is affected by light intensity, whereas the recombination rate is not.

$$
2 \mathrm{NOCl} \rightleftharpoons 2 \mathrm{NO}+\mathrm{Cl}_{2}
$$

An idealized photodiffusion membrane of total thickness $L$ consists of a dark region from $x=0$ to $L_{\mathrm{i}}$ and an illuminated region from $x=L_{\mathrm{i}}$ to $L$. The transport equations for this system in the dark or the illuminated part of the membrane can be written as:

$$
\begin{aligned}
& D_{\mathrm{A}} \frac{\mathrm{d}^{2} C_{\mathrm{A}}}{\mathrm{d} x^{2}}=r_{\mathrm{A}} \\
& D_{\mathrm{B}} \frac{\mathrm{d}^{2} C_{\mathrm{B}}}{\mathrm{d} x^{2}}=r_{\mathrm{A}} / 2 \\
& D_{\mathrm{AB}} \frac{\mathrm{d}^{2} C_{\mathrm{AB}}}{\mathrm{d} x^{2}}=-r_{\mathrm{A}}
\end{aligned}
$$


where $\mathrm{A}=\mathrm{NO}, \mathrm{B}=\mathrm{Cl}_{2}, \mathrm{AB}=\mathrm{NOCl}$, and $r_{\mathrm{A}}$ is the reaction rate in the dark or the illuminated part of the membrane. The reaction rate in the dark part of the membrane is given as:

$r_{\mathrm{A}}=k_{1} C_{\mathrm{A}}^{2} C_{\mathrm{B}}-k_{2} C_{\mathrm{AB}}^{2}$

In eqn. (3), $k_{1}$ and $k_{2}$ are the forward and the reverse rate constants for the reaction.

The reaction rate in the illuminated part of the membrane is given as:

$r_{\mathrm{A}}=k_{1} C_{\mathrm{A}}^{2} C_{\mathrm{B}}-k_{2} C_{\mathrm{AB}}^{2}-I_{\mathrm{a}}$

In eqn. (4), $I_{\mathrm{a}}$ is the contribution to the rate of reverse reaction due to the absorption of light. The boundary conditions are given as:

$\begin{array}{ll}C_{\mathrm{A}}=C_{\mathrm{A}}^{0} & \text { at } x=0 \\ C_{\mathrm{A}}=C_{\mathrm{A}}^{\mathrm{L}} & \text { at } x=L \\ \frac{\mathrm{d} C_{\mathrm{B}}}{\mathrm{d} x}=\frac{\mathrm{d} C_{\mathrm{AB}}}{\mathrm{d} x}=0 & \text { at } x=0 \text { and } L\end{array}$

In addition, the concentrations and the fluxes have to be continuous across the interface between the dark and the illuminated regions. This results in conditions similar to eqns. (6) for all the components:

$\left.C_{\mathrm{A}}\right|_{x=L_{\mathrm{i}}^{-}}=\left.C_{\mathrm{A}}\right|_{x=L_{\mathrm{i}}^{+}}$

and

$\left.\frac{\mathrm{d} C_{\mathrm{A}}}{\mathrm{d} x}\right|_{x=L_{\mathrm{i}}{ }^{-}}=\left.\frac{\mathrm{d} C_{\mathrm{A}}}{\mathrm{d} x}\right|_{x=L_{\mathrm{i}}{ }^{+}}$

The evaluation of the light absorption term in eqn. (4) can be accomplished using the Beer-Lambert law and is discussed in the Appendix. Light adsorption term, $I_{\mathrm{a}}$, can be written as:

$I_{\mathrm{a}}=I_{0} \epsilon_{\mathrm{av}} C_{\mathrm{AB}}\left(1-\epsilon_{\mathrm{av}} \int_{x}^{L} C_{\mathrm{AB}} \mathrm{d} x\right)$

In eqn. (7), $I_{0}$ is the light intensity incident on the membrane and $\epsilon_{\mathrm{av}}$ is the average molar absorptivity.

Equations (2)-(4) and (7), along with the boundary conditions given by eqns. (5) and (6), describe the light-assisted transport of NO across a membrane containing NOCl solution. The number of differential equations can be reduced to two by making a balance on the total amount of carrier (NOCl) charged into the membrane. For the present case, $D_{\mathrm{B}}$ and $D_{\mathrm{AB}}$ are equal (Table 1). It can be shown [12] that this condition along with the balance equation leads to:

$2 C_{\mathrm{B}}+C_{\mathrm{AB}}=C_{\mathrm{T}}$ 


\section{TABLE 1}

Values of physical parameters used in the calculations ${ }^{\mathrm{a}}$

Henry's law constant for NO, $H_{\text {NOFS-5 }}$

Equilibrium constant for $\mathrm{NOCl}$ decomposition reaction in dark, $K_{\mathrm{D}}$

Diffusion coefficient, $D_{\mathrm{NO}, \mathrm{FS}-5}$

Diffusion coefficient, $D_{\mathrm{NOC1}, \mathrm{FS}-5}$

Diffusion coefficient, $D_{\mathrm{Cl}_{2}, \mathrm{FS}-5}$
$1.5 \times 10^{5} \mathrm{~mol} / \mathrm{l}-\mathrm{mmHg}$

$4.35 \times 10^{8} \mathrm{l} / \mathrm{mol}$

$5.6 \times 10^{-6} \mathrm{~cm}^{2} / \mathrm{sec}$

$3.65 \times 10^{-6} \mathrm{~cm}^{2} / \mathrm{sec}$

$3.65 \times 10^{-6} \mathrm{~cm}^{2} / \mathrm{sec}$

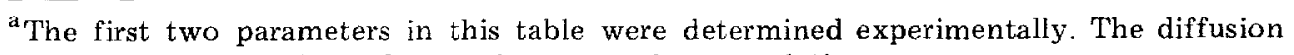
coefficients were estimated using the appropriate correlations.

In eqn. (8), $C_{\mathrm{T}}$ is the initial concentration of $\mathrm{NOCl}$. With this condition the equations for the illuminated zone of the membrane become:

$$
\begin{aligned}
& D_{\mathrm{A}} \frac{\mathrm{d}^{2} C_{\mathrm{A}}}{\mathrm{d} x^{2}}=\frac{1}{2} k_{1} C_{\mathrm{A}}^{2}\left(C_{\mathrm{T}}-C_{\mathrm{AB}}\right)-k_{2} C_{\mathrm{AB}}^{2} \\
& -I_{0} \epsilon_{\mathrm{av}} C_{\mathrm{AB}}\left(1-\epsilon_{\mathrm{av}} \int_{x}^{L} C_{\mathrm{AB}} \mathrm{d} x\right) \\
& D_{\mathrm{AB}} \frac{\mathrm{d}^{2} C_{\mathrm{AB}}}{\mathrm{d} x^{2}}=-\frac{1}{2} k_{1} C_{\mathrm{A}}^{2}\left(C_{\mathrm{T}}-C_{\mathrm{AB}}\right)+k_{2} C_{\mathrm{AB}}{ }^{2} \\
& +I_{0} \epsilon_{\mathrm{Av}} C_{\mathrm{AB}}\left(1-\epsilon_{\mathrm{av}} \int_{x}^{L} C_{\mathrm{AB}} \mathrm{d} x\right)
\end{aligned}
$$

The equations for the dark zone can be written similarly. A nearly "exact" solution of these integro-differential transport equations can be accomplished by numerical techniques but this is not a trivial task because these equations tend to be very stiff. The computer program developed by Jain and Schultz [12] can be used but would require a significant modification for this case. Also, since the reaction rate constants for NOCl decomposition in solvents are not known even approximately (only the equilibrium constant for $\mathrm{NOCl}$ decomposition in dark is known, as discussed later), meaningful comparisons between predictions and experiments would be difficult to make. Estimates for some limiting cases can, however, be obtained very easily without the knowledge of the individual kinetic parameters. One such limit is known as the "equilibrium-facilitation solution".

In the equilibrium-facilitation approximation to the solution it is assumed that the chemical reaction is in equilibrium everywhere in the membrane. In our experiments we measured concentrations of $\mathrm{NO}$ on both sides of the membrane under the conditions of no net flux. Based on our earlier calculations for the CO-hemoglobin system for no net flux conditions [11], 
it was thought that for $\mathrm{NOCl}$ system the "equilibrium-facilitation" estimate would provide a reasonable approximation for the actual experimental conditions. For $\mathrm{CO}$-hemoglobin system we showed that the total membrane thickness required to reach the equilibrium-facilitation limit for the no flux cases was less than $20 \mu \mathrm{m}$. Since the membrane thicknesses used in the present experiments were of the order of $600 \mu \mathrm{m}$, this solution was expected to be reasonably accurate.

\section{Equilibrium-facilitation solution of the transport equations}

Equations for the dark and the illuminated regions can be solved analytically for the reaction-equilibrium limit. Concentration ratio of the permeant (NO) across the membrane, supported by the light energy in the limit of zero flux, can also be determined. Flux equation for nitrix oxide can be derived starting with eqn. (2). Addition of equations for components $\mathrm{A}$ and $B$, and subsequent integrations along with the application of boundary conditions lead to:

$-N_{\mathrm{A}}=\frac{D_{\mathrm{A}}}{L}\left(C_{\mathrm{A}}^{0}-C_{\Lambda}^{L}\right)+\frac{D_{\mathrm{AB}}}{L}\left(C_{\Lambda \mathrm{B}}^{0}-C_{\mathrm{AB}}^{L}\right)$

where $C_{\mathrm{AB}}^{0}$ and $C_{\mathrm{AB}}^{L}$ are the concentrations of $\mathrm{NOCl}$ at $x=0$ and $x=L$, respectively. Equation (10) for the no flux case $\left(N_{\mathrm{A}}=0\right)$ reduces to:

$C_{\mathrm{AB}}^{L}=\frac{D_{\mathrm{A}}}{D_{\mathrm{AB}}}\left(C_{\mathrm{A}}^{0}-C_{\mathrm{A}}^{L}\right)+C_{\mathrm{AB}}^{0}$

Equations corresponding to the reaction-equilibrium state at $x=0$ and $x=L$ can be obtained by setting the reaction rates at these two boundaries equal to zero. The assumption of reaction-equilibrium at $x=0$ gives:

$\frac{1}{2} K_{\mathrm{D}}\left(C_{\mathrm{A}}^{0}\right)^{2}\left(C_{\mathrm{I}^{\prime}}-C_{\mathrm{AB}}^{0}\right)-\left(C_{\mathrm{AB}}^{0}\right)^{2}=0$

In eqn. (12), $K_{\mathrm{D}}$ is the equilibrium constant for the reaction in dark. Assuming reaction-equilibrium at $x=L$ gives:

$\frac{1}{2} k_{1}\left(C_{\mathrm{A}}^{L}\right)^{2}\left(C_{\mathbf{T}}-C_{\mathrm{AB}}^{L}\right)-k_{2}\left(C_{\mathrm{AB}}^{L}\right)^{2}-I_{0} \epsilon_{\mathrm{aV}} C_{\mathrm{AB}}^{L}=0$

The experimental results of Jain [13] suggest that the contribution of thermal decomposition to the reverse rate is likely to be much smaller compared to the contribution from the photochemical decomposition. Use of this approximation and the rearrangement of the resulting equation gives:

$\frac{I_{0} \epsilon_{\mathrm{av}}}{k_{1}}=\left(C_{\mathrm{A}}^{L}\right)^{2}\left(C_{\mathrm{T}}-C_{\mathrm{AB}}^{L}\right) / 2 C_{\mathrm{AB}}^{L}$

Equations (11), (12), and (14) can be used to determine the effect of light 
intensity on the facilitation ratio, $\left(C_{\mathrm{A}}^{L} / C_{\mathrm{A}}^{0}\right)$, if the left side in eqn. (14) and $C_{\mathrm{A}}^{0}$ are known.

\section{Experimental}

The experimental procedure for studying the light-assisted transport of nitric oxide across the membranes involved illuminating an organic solution of nitrosyl chloride contained in a membrane sandwich exposed to equal concentrations of nitric oxide on both sides. Each experiment was continued until the nitric oxide concentrations on both sides of the membrane did not change with time. At this point gas samples on the upstream and the downstream sides of the membrane sandwich were analyzed using ion-selective electrodes to determine the effect of various variables on nitric oxide facilitation.

\section{Materials and equipment}

Special materials of construction and special handling procedures had to be used because of the corrosive and toxic nature of nitrosyl chloride and the reaction products. The materials of construction included glass, teflon and nylon 6,6 for the structural parts of the equipment and viton, teflon and FETFE were used as the O-ring materials. Glass stopcocks with teflon and FETFE O-rings were used throughout the system. The organic solvent used in the experiments to dissolve NOCl was a perhalogenated solvent called FS-5 (tradename of Hooker Chemical Co., Niagara Falls, NY) with a chemical formula denoted as $\left(\mathrm{C}_{2} \mathrm{~F}_{3} \mathrm{Cl}\right)_{n}$. This solvent was chosen after initial tests with a number of solvents because of its very low vapor pressure, high $\mathrm{NOCl}$ and $\mathrm{Cl}_{2}$ solubility and low NO solubility.

Nitrosyl chloride gas used in the experiments was obtained in the lecture bottle form. Certified nitric oxide mixtures in $\mathrm{N}_{2}(0.1$ and $1.0 \%)$ and high purity $\mathrm{N}_{2}(99.998 \%)$ were used in transport studies. The optical system for the illumination of the membrane sandwich was obtained from Oriel Corp. (Stratford, CT). It included an air-cooled universal lamp housing with $f / 1.5$ fused silica condensing lens, a $150 \mathrm{~W}$ xenon arc lamp, a liquid filter assembly, metallic neutral density filters with N.D.'s of $0.1,0.3,0.5$ and 1.0 , and a $90^{\circ}$ light tube.

The equipment used in the analysis with ion-selective electrodes (model 94-17 chloride electrode, model 97-70 residual chlorine electrode, model 90-02 double junction reference electrode and model $801 \mathrm{mV} / \mathrm{pH}$ meter) was obtained from Orion Research Inc. (Cambridge, MA). All the chemicals used in the analysis with ion-selective electrodes were of analytical grade. Various membranes used in the experiments were: polyester laminated silicone rubber membranes (General Electric Co.; Schenectady, NY), white nitrocellulose membranes (Nucleopore Corp.; Pleasanton, CA), and black cellulose and white polyvinylidene fluoride (PVDF) membranes (Millipore Corp.; Bedford, MA). Cellulose and PVDF membranes had nominal pore 
sizes and thicknesses of $0.45 \mu \mathrm{m}$ and $150 \mu \mathrm{m}$, respectively, while silicone rubber membrane had a nominal thickness of $25 \mu \mathrm{m}$.

Details of the experimental system and the experimental procedure

The experimental set up for the membrane transport experiments is shown in Fig. 1. The heart of the experimental apparatus was a nylon 6,6 diffusion cell constructed in two halves. The diffusion cell contained a precision-machined cavity for the membrane sandwich and a viton O-ring was used to seal the two halves of the diffusion cell. The top half of the diffusion cell contained one inlet port for the NOCl solution and one outlet port for the sample removal. The bottom half contained an inlet port for the nitric oxide mixture. The membrane sandwich was illuminated through a quartz window in the top half of the cell. The diffusion cell was submerged in a plexiglas constant temperature bath. The volume on the downstream side (about $1000 \mathrm{ml}$ ) was much larger compared to the volume on the upstream side. This kept the downstream concentration nearly constant during the experiment. Proper recirculation was provided on both sides of the diffusion cell. The light from the xenon arc lamp was filtered through a $10 \mathrm{~cm}$ long distilled water column to remove the infrared component of the light. Neutral density filters were interposed in the path of light to change the relative light intensity. The experiments were carried out inside a fume hood.

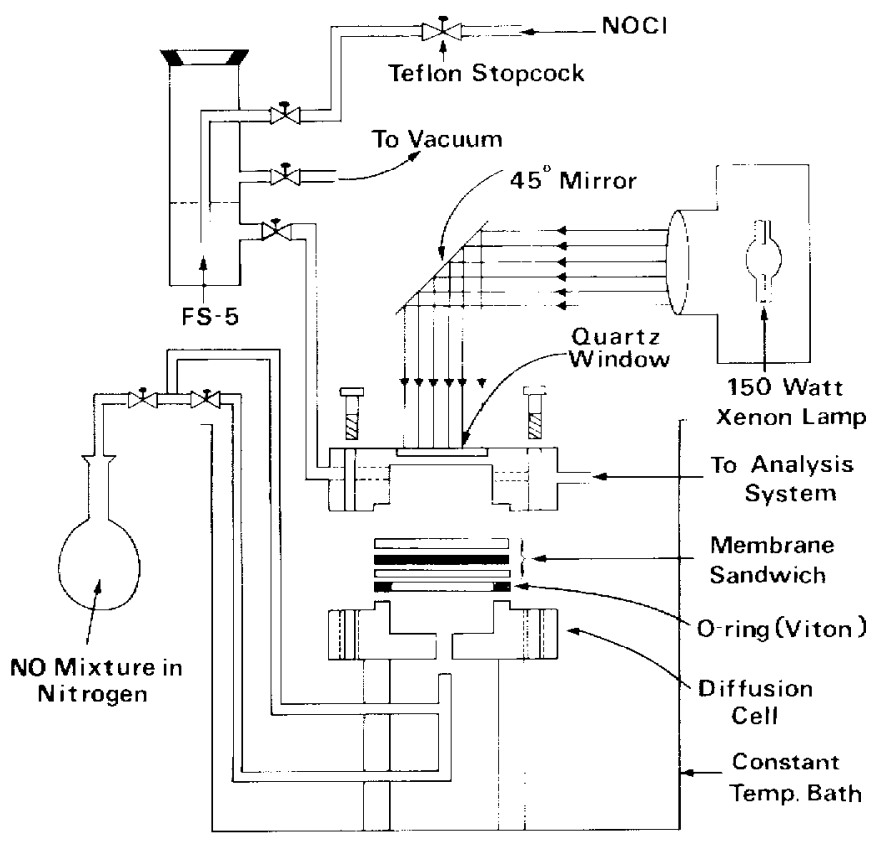

Fig. 1. Schematic diagram of the experimental set up for the membrane transport experiments. 
At the start of each experiment a membrane sandwich consisting of (from top): white PVDF membrane, black cellulose membrane, white cellulose membrane, silicone rubber membrane and a thick porous polyethylene membrane, was clamped between the two halves of the diffusion cell. The white PVDF membrane (which becomes transparent in the NOCl solution) served as the illuminated side in the photomembrane configuration. The black and the white cellulose membranes served as the opaque barrier and the dark side respectively. The silicone membrane (which was not wetted by FS-5) was used to prevent the NOCl solution from falling through the membranes. The porous polyethylene membrane was used as a support for the membrane sandwich.

The next step in the experimental procedure involved the evacuation of the entire apparatus including the flask containing FS-5 solution to remove all traces of oxygen which reacts irreversibly with nitric oxide. Both upstream and downstream sides of the diffusion cell were filled with nitric oxide mixture in $\mathrm{N}_{2}$. The nitrosyl chloride solution was prepared in the flask containing FS-5 and was transferred to the diffusion cell via a capillary. The diffusion cell containing $\mathrm{NOCl}$ solution was allowed sufficient time to reach the bath temperature.

Finally, the membrane sandwich containing NOCl solution was illuminated allowing enough time for the attainment of the photostationary state. In the photostationary state, the net flux of nitric oxide across the membrane becomes zero and a higher concentration of nitric oxide on the upstream side compared to the downstream side is established. After the end of each experiment, known volumes of the samples on the upstream and the downstream sides of the diffusion cell were dissolved in phosphate buffer and were analyzed to find partial pressures of various gases on the upstream and the downstream side.

\section{Analytical procedure}

The concentrations of various gases in the gas phase (NOCl, $\mathrm{NO}$, and $\mathrm{Cl}_{2}$ ) were determined using a combination of ion-selective electrodes. These gases when dissolved in the phosphate buffer solution produce nitrite $\left(\mathrm{NO}_{2}\right)$, chloride $\left(\mathrm{Cl}^{-}\right)$, nitrate $\left(\mathrm{NO}_{3}^{-}\right)$and hypochlorite $\left(\mathrm{OCl}^{-}\right)$ions. The concentrations of nitrite, 'chloride and the hypochlorite ions were found using a nitric oxide electrode, a solid-state chloride electrode and a residual chlorine electrode, respectively. Nitrate ion concentration was also found with a nitric oxide electrode after reducing nitrate to nitrite using a copperized-cadmium column. All the ions were present at low levels in the solution and for accurate measurement of these concentrations a nonlinear calibration method proposed by Jain and Schultz [14] was used. This method results in errors of less than $5 \%$ in the concentration measurements. Once the ion concentrations had been determined, they were related to the partial pressures of the individual gases in the gas phase [13]. Nitrosyl chloride concentration in FS-5 was determined by extracting a small portion of organic solution in 
phosphate buffer and measuring the chloride ion concentration in the resulting solution.

\section{Results and discussion}

The effect of light intensity on the decomposition of nitrosyl chloride solution in FS-5 was studied before membrane transport experiments. The membrane transport experiments were carried out by varying nitric oxide (permeant) concentration, nitrosyl chloride (carrier-complex) concentration and the light intensity. The results discussed include the effect of these variables on the facilitation ratio of nitric oxide across the membrane under no flux condition. The results can be described reasonably well using the "equilibrium-facilitation" model.

Effect of light intensity on the decomposition of nitrosyl chloride solutions

The effect of light intensity on $\mathrm{NOCl}$ decomposition was studied by illuminating $\mathrm{NOCl}$ solution contained in a jacketed photoreactor. After establishing the photostationary state, the partial pressures of various gases in the gas phase were measured. These were used to determine liquid phase concentrations of various gases. The details of the experimental procedure and the analysis are discussed by Jain [13]. The liquid phase concentrations can be used to calculate $K_{\mathrm{L}}$

$K_{\mathrm{L}}=[\mathrm{NOCl}]^{2} /[\mathrm{NO}]^{2}\left[\mathrm{Cl}_{2}\right]$

which at $0 \%$ light intensity is a measure of the equilibrium constant for $\mathrm{NOCl}$ decomposition in dark. The ratio of the value of $K_{\mathrm{L}}$ at other light intensities to its value in dark provides a measure of the effect of light on $\mathrm{NOCl}$ decomposition. The values of $K_{\mathrm{L}}$ at different light intensities are plotted in Fig. 2. The results show that the value of $K_{\mathrm{L}}$ changes by a factor of over 200 as the relative light intensity is increased from 0 to $100 \%$. This indicates that the decomposition of $\mathrm{NOCl}$ can be increased significantly by increasing the light intensity.

\section{Summary of the membrane transport experiments}

Absolute light intensities could not be measured in our experiments and only relative values are reported. Most experiments were carried out at low level of relative light intensity to minimize the heating effect of light. Initial experiments were conducted at room temperature $\left(25^{\circ} \mathrm{C}\right)$ using $1 \%$ nitric oxide mixture in $\mathrm{N}_{2}$. The results for these experiments are shown in Fig. 3. At a $\mathrm{NOCl}$ concentration of about $0.14 \mathrm{M}$, concentration ratios of NO of up to 3 were obtained. At a slightly lower NOCl concentration $(0.10 M)$, concentration ratios of up to 2.5 were obtained. The concentration effect was found to increase with the increase in light intensity. In these experiments it was found that up to $40 \%$ of total gas phase consisted of NOCl. This suggests a substantial carrier loss at this temperature. This behavior 

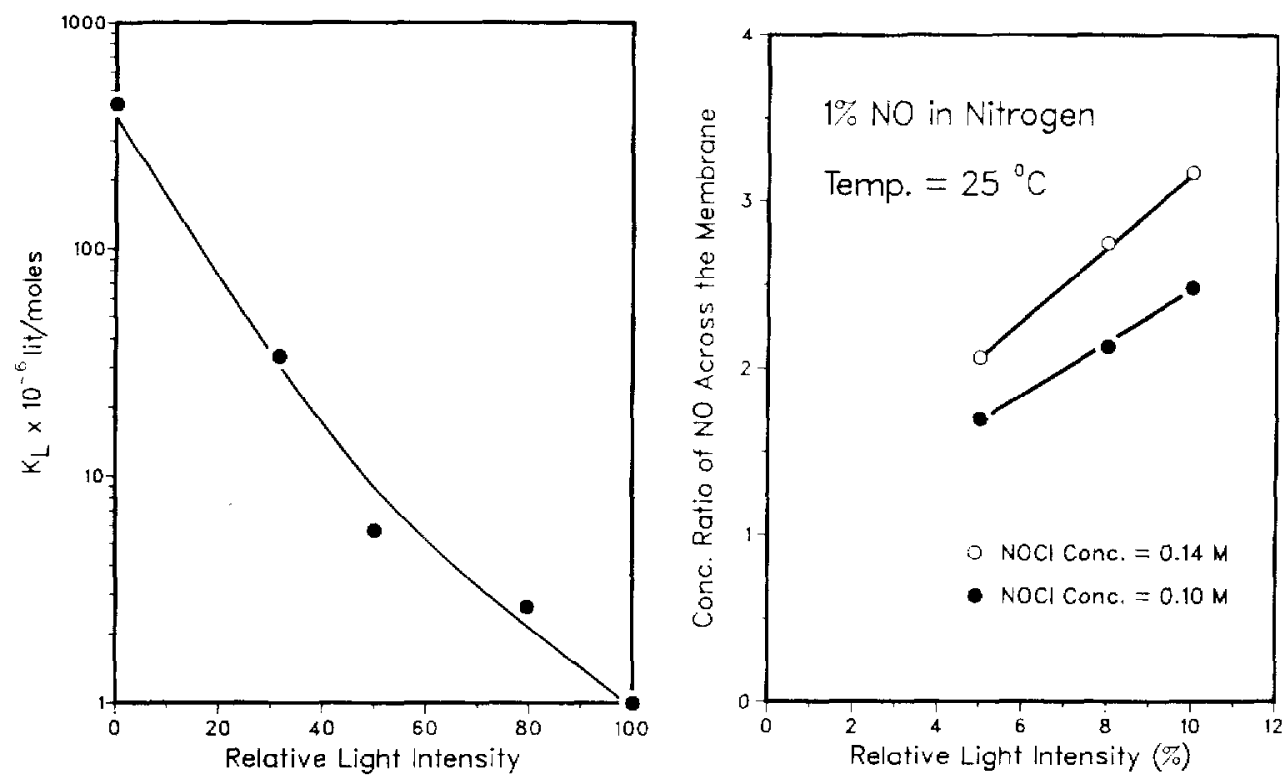

Fig. 2. Effect of light intensity on the decomposition of nitrosyl chloride solutions.

Fig. 3. Facilitation ratio vs. light intensity results for NO transport across membranes containing $\mathrm{NOCl}$ solutions.

may result from high vapor pressure of $\mathrm{NOCl}$ at room temperature or due to the heating effect of light.

Further experiments were conducted at a temperature close to $2^{\circ} \mathrm{C}$ using the ice water in the bath. Some trial experiments in presence of light, using $\mathrm{NOCl}$ solution in the membrane sandwich and pure $\mathrm{N}_{2}$ on both sides, indicated that NOCl loss was likely to be much smaller at this temperature. The results of the low temperature experiments are shown in Figs. 4 and 5.

The experiments reported in Fig. 4 were conducted using $1 \%$ NO mixture in nitrogen. It can be seen that facilitation ratios of close to 5 were obtained in these experiments at a $\mathrm{NOCl}$ concentration of about $0.26 \mathrm{M}$. At a slightly lower $\mathrm{NOCl}$ concentration, facilitation ratios of up to 4 were obtained. The amount of $\mathrm{NOCl}$ in the gas phase was less than $10 \%$ in these experiments.

In Fig. 5, the facilitation results obtained with $0.1 \% \mathrm{NO} / \mathrm{N}_{2}$ mixture are shown. The facilitation ratios obtained here are significantly higher compared to the facilitation ratios obtained with $1 \% \mathrm{NO} / \mathrm{N}_{2}$ mixture. At a $\mathrm{NOCl}$ concentration of about $0.14 \mathrm{M}$ and a relative light intensity of $32 \%$, a facilitation ratio close to 15 was obtained. A facilitation ratio close to 9 was obtained at $10 \%$ relative light intensity and $\mathrm{NOCl}$ concentration of $0.183 \mathrm{M}$. At low values of relative light intensity the facilitation ratios are very close at two different $\mathrm{NOCl}$ concentrations. The difference increases 
with the increase in relative light intensity. The results of the experiments in Figs. 4 and 5 were analyzed using the equilibrium-facilitation model discussed earlier in this paper. The physical constants needed in the equilibrium-facilitation model were either found experimentally or estimated empirically [13]. The values are listed in Table 1.
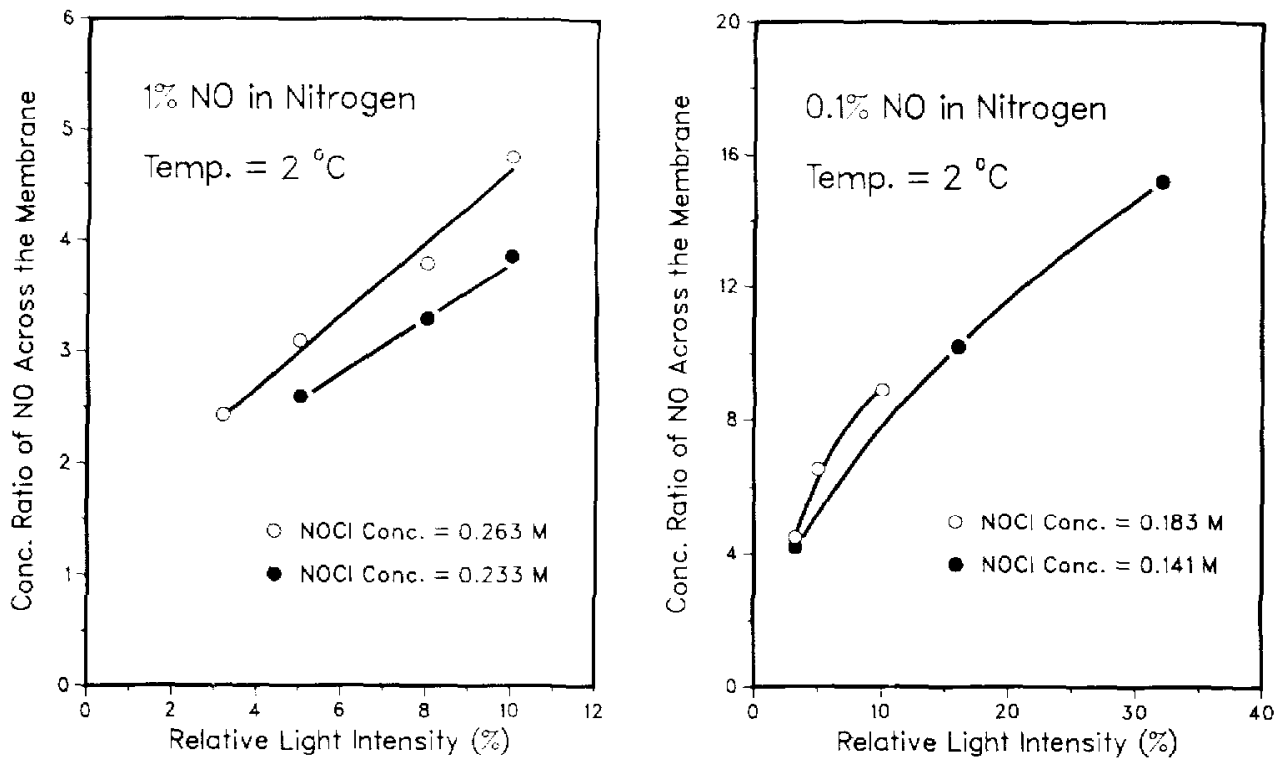

Fig. 4. Effect of light intensity on nitric oxide facilitation ratio across membranes con taining $\mathrm{NOCl}$ solutions (Temp. $=2 \mathrm{C}, 1 \% \mathrm{NO}$ in $\mathrm{N}_{2}$ ).

Fig. 5. Effect of light on nitric oxide facilitation ratio (Temp. $=2^{\circ} \mathrm{C}, 0.1 \% \mathrm{NO}$ in $\mathrm{N}_{2}$ ).

\section{Analysis of the results}

For each experiment, the concentration of NO on both sides of the membrane, relative light intensity, and the $\mathrm{NOCl}$ concentration are known. Using the known values of $K_{\mathrm{D}}, C_{\mathrm{A}}^{0}$ and $C_{\mathrm{T}}$ in eqn. (12), the value of the carrier-complex concentration at $x=0, C_{\mathrm{AB}}^{0}$, can be calculated. Once the value of $C_{\mathrm{AB}}^{0}$ is known, it can be used in eqn. (11) to calculate $C_{\mathrm{AB}}^{L}$. Substitution of various values in the right hand side of eqn. (14) enables the evaluation of $I_{x}=0 \epsilon_{\mathrm{av}} / k_{1}$. Dividing this quantity by $I_{\mathrm{R}}$, relative light intensity, gives the value of $I_{0} c_{\mathrm{av}} / k_{1}$ for each experiment. If the equilibriumfacilitation model is valid, the values of $I_{0} \epsilon_{\mathrm{av}} / k_{1}$ obtained from different experiments should be the same.

The values of $I_{0} \epsilon_{\mathrm{av}} / k_{1}$ calculated from the experimental data for various experiments are plotted in Fig. 6. It can be seen that the values from different experiments do not differ significantly from each other. A mean value of $I_{0} \epsilon_{\mathrm{av}} / k_{1}=9.0 \times 10^{-8} \mathrm{~mol}^{2} / 1^{2}$ and a maximum deviation from the mean of about $30 \%$ were found. This provides at least a partial verification of the equilibrium-facilitation analysis used to analyze the results. 


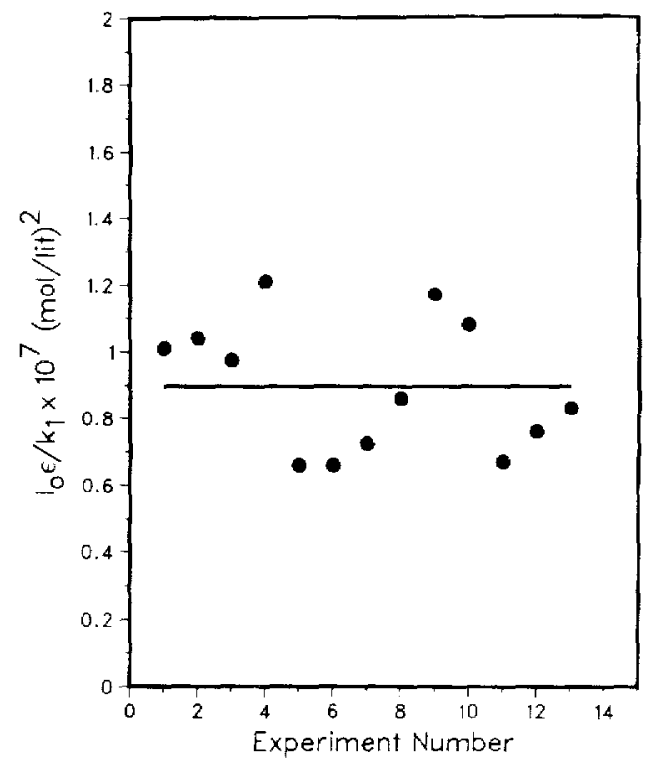

Fig. 6. Summary of the results on NO facilitation.

\section{Conclusions}

In this paper it was experimentally demonstrated that nitric oxide can be concentrated using light energy as the driving force. This is accomplished by differentially illuminating a membrane sandwich containing nitrosyl chloride solution. The concentration ratios of nitric oxide supported across the membrane depend on the light intensity, nitrosyl chloride concentration, and nitric oxide concentration. Concentration ratios ranging from 2 to 15 were oblained depending on the combination of various parameters. The results were explained using a theory which assumes the chemical reaction to be in equilibrium everywhere in the membrane. The concentration gradients obtained in these experiments can be coupled to a fuel cell as discussed by Jain and Schultz [11] to convert chemical potential gradient to electrical energy. When the light used for the illumination is sun light, the system can be used for the storage of solar energy in electrical form.

\section{Acknowledgement}

This research was supported by NSF Grant No. NSF-CPE 78-13316. The typing assistance of D. Vickery and M. Frey is gratefully acknowledged.

\section{List of symbols}

A Nitric oxide (NO) 


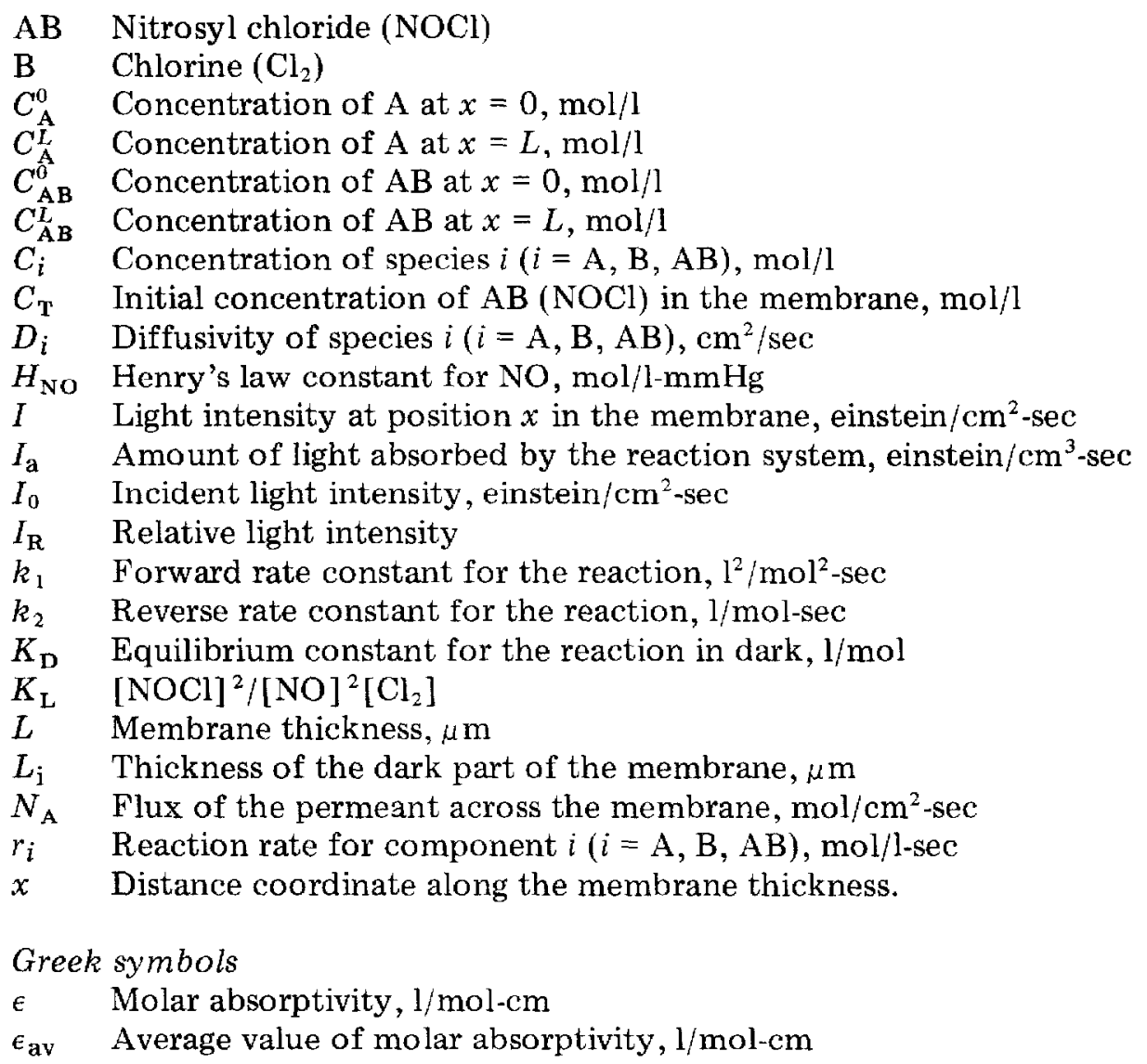

\section{References}

1 G.B. Kistiakowsky, Photochemical decomposition of nitrosyl chloride, J. Amer. Chem. Soc., 52 (1930) 102.

2 G.L. Natanson, On the mechanism of photochemical decomposition of nitrosyl chloride, Acta Physiochim. U.R.S.S., II (1939) 521.

3 K. Atwood and G.K. Rollefson, The efficiency of the primary photolysis process in the solution, J. Chem. Phys., 9 (1941) 506.

4 O.S. Newirth, The photolysis of nitrosyl chloride and the storage of solar energy, J. Phys. Chem., 63 (1959) 17.

5 R.J. Marcus and H.C. Wohlers, Photolysis of nitrosyl chloride in a solar furnace, Sol. Energy, 4 (1960) 1.

6 R.J. Marcus and H.C. Wohlers, Photochemical systems for solar energy conversion Nitrosyl chloride, Sol. Energy, 5 (1961) 44.

7 W.E. McKee, E. Findl, J.D. Margerum and W.B. Lee, A nitrosyl chloride solar regenerative fuel cell system, WADD Tech. Rep. 60-821, Part I, 1960.

8 R.J. Marcus and H.C. Wohlers, Flow systems in the solar furnace and the photolysis of nitrosyl chloride, Sol. Energy, 5 (1961) 121. 
9 W.E. McKee, E. Findl, J.D. Margerum and W.B. Lee, A nitrosyl chloride solar regenerative fuel cell system, WADD Tech. Rep. 60-821, Part II, 1961.

10 J.S. Schultz, Carrier-mediated photodiffusion membranes, Science, 197 (1977) 1177.

11 R. Jain and J.S. Schultz, An analysis of carrier-mediated photodiffusion membranes, J. Membrane Sci., 15 (1983) 63.

12 R. Jain and J.S. Schultz, A numerical technique for solving carrier-mediated transport problems, J. Membrane Sci., 11 (1982) 79.

13 R. Jain, Light-assisted transport of gases across supported liquid membranes, Ph.D. Thesis, The University of Michigan, Ann Arbor, MI, U.S.A., 1984, Chaps. IV and V.

14 R. Jain and J.S. Schultz, Data analysis for concentration measurements in the nonlinear response region of ion-selective electrodes, Anal. Chem., 56 (1984) 141.

15 J.G. Calvert and J.N. Pits, Photochemistry, Wiley, New York, NY, 1966, pp. 640642.

16 C.F. Goodeve and S. Katz, The absorption spectrum of nitrosyl chloride, Proc. Roy. Soc. London Ser. A, 172 (1939) 432.

\section{Appendix}

Evaluation of the light absorption term in eqn. (4)

Beer-Lambert law is used for the evaluation of light absorption term. If $I_{0}$ is the light intensity incident on the membrane system (einstein $/ \mathrm{cm}^{2}$. sec), the light intensity variation as a function of membrane depth can be calculated using eqn. (A.1):

$I=I_{0} \exp \left(-\int_{x}^{L} \epsilon C_{\mathrm{AB}}(x) \mathrm{d} x\right)$

In eqn. (A.1), $C_{\mathrm{AB}}(x)$ is the NOCl concentration at position $x$ in the membrane and $\epsilon$ is its molar absorptivity. The absorption of light by NO and $\mathrm{Cl}_{2}$ is small compared to the absorption by $\mathrm{NOCl}$ and can be neglected. Using eqn. (A.1), it can be shown [15] that the light absorption term, $I_{\mathrm{a}}$, is given by:

$I_{\mathrm{a}}=I_{0} \in C_{\mathrm{AB}} \exp \left(-\int_{x}^{L} \epsilon C_{\mathrm{AB}} \mathrm{d} x\right)$

Since the molar absorptivity depends on the wavelength, ideally one should use equations similar to eqn. (A.2) for each wavelength and add individual contributions to get the total amount of light absorbed. However, in our appratus the light output from the light source does not vary significantly with wavelength in the wavelength interval for absorption [13] and an average value of $\epsilon$ over the interval for absorption ( $350 \mathrm{~nm}$ to 615 $\mathrm{nm}$ ) can be used. Using this approximation and denoting the average value of molar absorptivity as $\epsilon_{a \mathrm{av}}$, eqn. (A.2) can be rewritten as:

$I_{\mathrm{a}}=I_{0} \epsilon_{\mathrm{av}} C_{\mathrm{AB}} \mathrm{e}^{-y}$ 
where

$y=\epsilon_{\mathrm{av}} \int_{x}^{L} C_{\mathrm{AB}} \mathrm{d} x$

Average value of the molar absorptivity in eqn. (A.3) can be calculated using data from Goodeve and Katz [16]. A numerical integration of Goodeve and Katz data in the wavelength interval 350 to $615 \mathrm{~nm}$ gave an average value of $7.0 \mathrm{l} / \mathrm{mol}-\mathrm{cm}$ for $\epsilon$. In our experiments, the maximum value of $L-L_{\mathrm{i}}$, the thickness of the illuminated zone of the membrane, was $500 \mu \mathrm{m}$ and the maximum $\mathrm{NOCl}$ concentration in the membrane was 0.3 $\mathrm{mol} / \mathrm{l}$. Using these values it can be shown [13] that the maximum error in approximating $e^{-y}$ in eqn. (A.3) by $(1-y)$ is less than $1 \%$. With this approximation, eqn. (A.3) can be rewritten as:

$I_{\mathrm{a}}=I_{0} \epsilon_{\mathrm{av}} C_{\mathrm{AB}}\left(1-\epsilon_{\mathrm{av}} \int_{x}^{L} C_{\mathrm{AB}} \mathrm{d} x\right)$ 